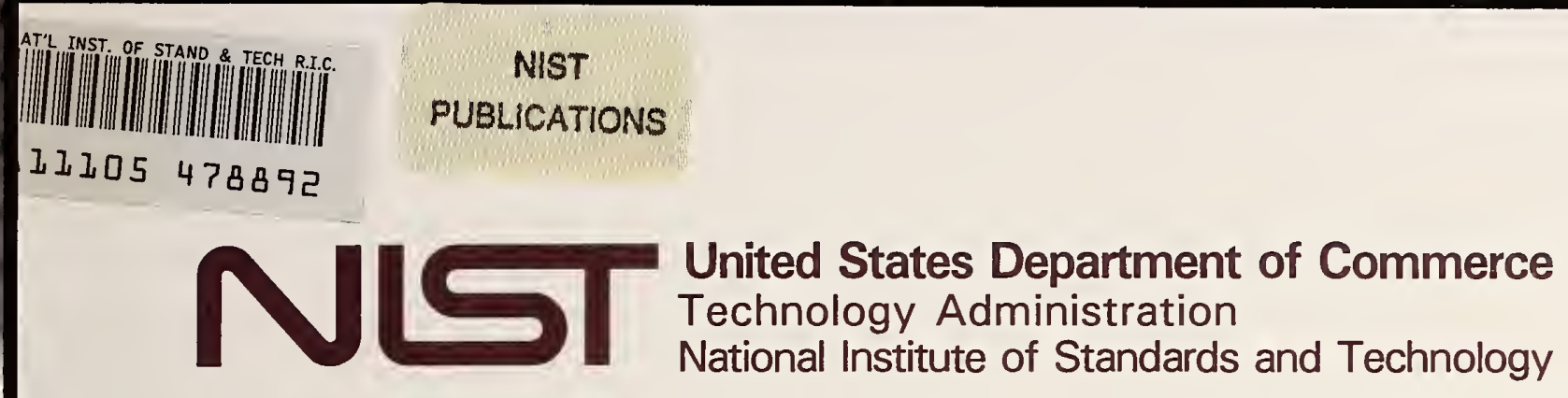

NIST Technical Note 1505

\title{
Stripline Resonator for Electromagnetic Measurements of Materials
}

Chriss A. Jones

Yehuda Kantor John H. Grosvenor Michael D. Janezic

jC

00

15753

10.1505

998

C. 2 

NIST Technical Note 1505

\section{Stripline Resonator for Electromagnetic Measurements of Materials}

Chriss A. Jones

Yehuda Kantor

John H. Grosvenor

Michael D. Janezic

Electromagnetic Fields Division

Electronics and Electrical Engineering Laboratory

National Institute of Standards and Technology

325 Broadway

Boulder, Colorado 80303-3328

July 1998

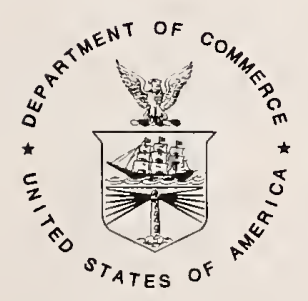

U.S. DEPARTMENT OF COMMERCE, William M. Daley, Secretary 
National Institute of Standards and Technology Technical Note Natl. Inst. Stand. Technol., Tech. Note 1505, 24 pages (July 1998) CODEN:NTNOEF

U.S. GOVERNMENT PRINTING OFFICE

WASHINGTON: 1998

For sale by the Superintendent of Documents, U.S. Government Printing Office, Washington, DC 20402-9325 


\title{
Stripline Resonator for Electromagnetic Measurements of Materials*
}

\author{
Chriss A. Jones*, Yehuda Kantor ${ }^{\dagger}$, \\ John H. Grosvenor*, Michael D. Janezic*
}

\begin{abstract}
A stripline resonator was used to measure the relative dielectric and magnetic properties of materials in the frequency range from $150 \mathrm{MHz}$ to $2 \mathrm{GHz}$. Measurement accuracy relates strongly to the dimensions of the resonator. Consequently, equations used in the design of a resonator and the resonator design are described and design tradeoffs are discussed. Dielectric and magnetic measurements are included to support the design of our resonator. Discussions of important concepts used in measurement correction, such as sample shape and size and demagnetization, are provided.
\end{abstract}

Keywords: ferrite; loss tangent; permittivity; permeability; stripline; resonator.

\section{INTRODUCTION}

The stripline resonator cavity was developed by Waldron and Maxwell [1-4], to study the permittivity and permeability of ferrites at low microwave frequencies and to overcome difficulties associated with the more common coaxial transmission line technique. A stripline resonator consists of two ground planes and center conductor as shown in Figure 1. The open structure of the resonator allows the sample to be placed inside and removed from the cavity easily without breaking circuit connections. This structure also allows an external magnetic field to be applied in order to measure the gyromagnetic ratio [5]. Another benefit is that the sample is rectangular instead of toroidal which is easier to machine and allows tighter machining tolerances. Although transmission line techniques are applicable over a broad frequency range, resonator techniques are more sensitive and allow small samples of low- or high-loss sample material.

The stripline resonator is an open structure and does not allow analytic solution of the fields. Instead a conformal mapping is used to determine the field structure. This field structure allows us to

"RF Technology Division, MS 813.01, National Institute of Standards and Technology, Boulder, CO 80303-3328.

${ }^{\dagger}$ RAFAEL, Electric Systems Division, Dept. 87, P.O. Box 2250, Haifa, Israel. 


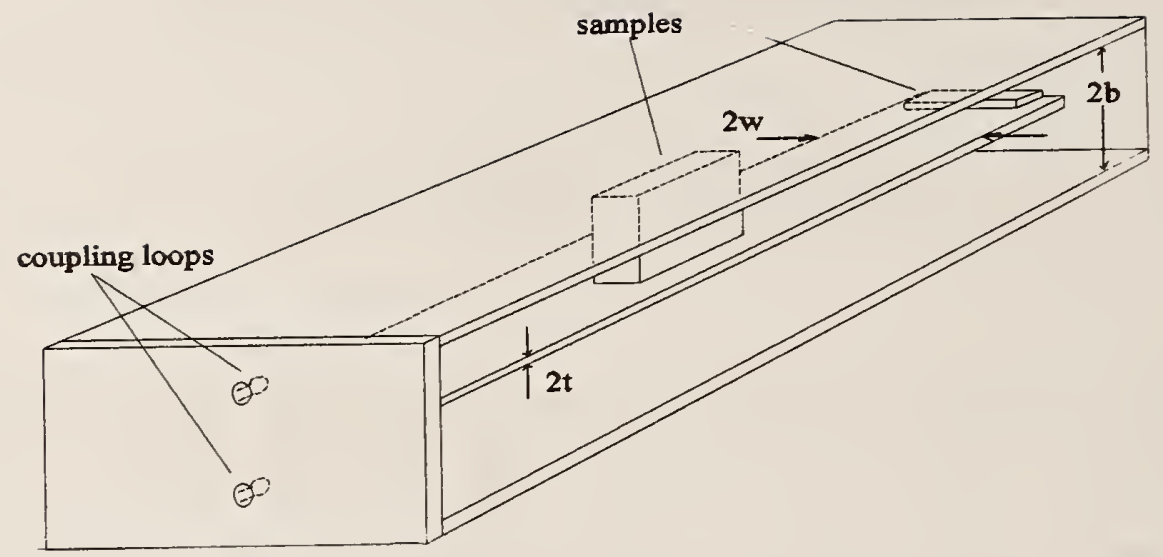

Figure 1. Stripline Resonator showing placement of samples for measurement of permittivity and permeability

determine the dimensions of the resonator and to develop a perturbation equation used to determine the permittivity $\left(\epsilon^{*}=\epsilon^{\prime}-\mathrm{j} \epsilon^{\prime \prime}\right)$ and permeability $\left(\mu^{*}=\mu^{\prime}-\mathrm{j} \mu^{\prime \prime}\right)$ of a material sample. The design of the resonator contributes to the uncertainty in the measurement results and it is the intent of this paper to discuss these design criteria and to show that accurate results can be obtained using the appropriate corrections.

Permittivity measurements are made by placing the sample in a region of maximum electric field (zero magnetic field) to allow for a maximum perturbation of the fields, and permeability is measured by placing the material in a region of maximum magnetic field (zero electric field). If a dielectric sample does not fill the space between the conductors, a depolarization occurs [6]. A similar effect occurs for the magnetic sample since the sample does not surround the center conductor. The electric or magnetic field in the cavity induces surface electric or magnetic dipole moments in the material which set up a field inside the material that opposes the applied field. Thus when you measure the relative dielectric or magnetic properties of materials in the stripline resonator, lower values of permittivity or permeability $\left(\epsilon_{\mathrm{m}}^{\prime}, \epsilon_{\mathrm{m}}^{\prime \prime}, \mu_{\mathrm{m}}^{\prime}\right.$ and $\left.\mu_{\mathrm{m}}^{\prime \prime}\right)$ are measured rather than the true intrinsic relative properties $\left(\epsilon_{\mathrm{i}}^{\prime}, \epsilon_{\mathrm{i}}^{\prime \prime}, \mu_{\mathrm{i}}^{\prime}\right.$ and $\left.\mu_{\mathrm{i}}^{\prime \prime}\right)$. All references to permittivity and permeability from here on will be relative values.

Section 2 discusses background information on the mapping functions and the equations used to determine the dimensions of the resonator. A discussion of the perturbation equation used to determine the permittivity and permeability is also presented. The ideas behind depolarization [6] and demagnetization [7-11,14] are introduced in this section and will provide us with the means to correct measurement results. Section 3 is used as a guide in the design of an example resonator and the 
dimensions of our resonator are presented as well. Pitfalls are discussed. Section 4 supports the design by presenting measurement results using the demagnetization correction.

\section{THEORETICAL BACKGROUND}

\subsection{Design Equations}

In order to predict the electromagnetic field behavior with and without the sample, Waldron $[1,3]$ used a theoretical prediction of the mapping function from the stripline cavity to a parallel-plate cavity. The mapping functions have two parameters, $\alpha>1$ and $\beta<1$, that can be freely chosen due to the nature of Schwarz-Christoffel mappings. These parameters describe the uniformity of the field lines between the center conductor and ground planes. Figure 2 shows the electric and magnetic field line structure in the cross section of the stripline resonator. Line $Q R$ is the electric field line in the center of the cavity, line $A B$ is the electric field line at the end of the sample, and point $P$ is assumed to map to infinity but can be considered to be at the end of the ground planes. As $\alpha$ and $\beta$ approach 1 , line $A B$ will be parallel to line $Q R$, so the entire sample will be in a uniform electric field. The following are formulas for the main geometrical dimensions of the stripline resonator derived from the mapping function [1]. These dimensions are related to the parameters $\alpha$ and $\beta$ through the mapping functions. The importance of the relations will be demonstrated with a numerical example given in Section 3.1 .

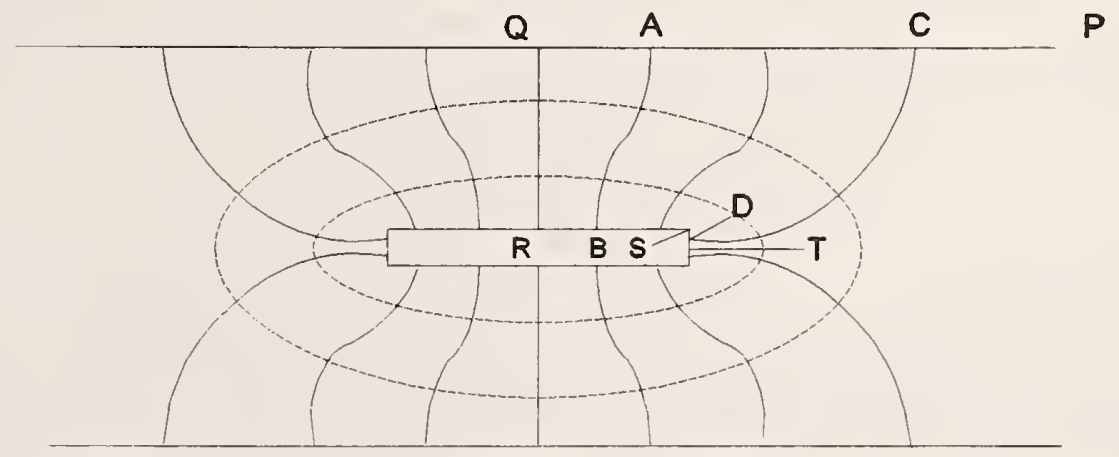

Figure 2. Electric and magnetic field structure for the stripline resonator shown in cross-section 
The half thickness $t$ of the center conductor is given by

$$
t=-\lambda\left[\mathrm{K}\left(\beta^{\prime}\right)-\frac{\alpha^{2}-\beta^{2}}{\alpha^{2}-1} \Pi\left(\frac{1-\beta^{2}}{1-\alpha^{2}}, \beta^{\prime}\right)\right],
$$

where $\mathrm{K}$ is the complete elliptic integral of the first kind, $\Pi$ is the complete elliptic integral of the third kind, $\alpha$ and $\beta$ are free parameters to be chosen, and $\beta^{\prime}$ is the complementary modulus of $\beta$. $\lambda$ has dimensions of half the separation $b$, between ground planes and is related to $\alpha$ and $\beta$ by

$$
\lambda=\frac{2 \alpha b}{\pi} \sqrt{\frac{\alpha^{2}-1}{\alpha^{2}-\beta^{2}}} .
$$

The half width $w$ of the center conductor is given by

$$
w=\lambda\left[\mathrm{K}(\beta)-\left(1-\frac{\beta^{2}}{\alpha^{2}}\right) \Pi\left(\frac{\beta^{2}}{\alpha^{2}}, \beta\right)\right],
$$

and the half separation $b$ between the ground planes is given by

$$
b=\lambda\left(\frac{\alpha^{2}-\beta^{2}}{\alpha^{2}-1}\right)\left[\Pi\left(\frac{1-\beta^{2}}{1-\alpha^{2}}, \beta\right)+\Pi\left(1-\alpha^{2}, \beta^{\prime}\right)-\mathrm{K}\left(\beta^{\prime}\right)\right] .
$$

These equations allow us to map the field lines from the stripline resonator as shown in Figure 2 to the field lines in a parallel-plate cavity. From this mapping, the dimensions of the resonator can be chosen by plotting the ratio $t / b$ of the thickness to separation and the ratio $w / b$ of width to separation versus $\alpha$ and $\beta$. These plots are shown in Figures 6 and 7 of Section 3 .

\subsection{Perturbation Theory}

The expressions for the change in quality factor and frequency shift of the resonator, due to insertion of the sample, lead to equations for the permittivity and permeability. This is based on the wellknown perturbation equation [12],

$$
\frac{\delta \omega}{\omega}+\frac{\mathrm{j}}{2 Q_{0}}=\frac{\iiint_{V_{1}}\left[\left(E_{1} \cdot D_{0}-E_{0} \cdot D_{1}\right)-\left(H_{1} \cdot B_{0}-H_{0} \cdot B_{1}\right)\right] d V}{\iiint_{V_{0}}\left(E_{0} \cdot D_{0}-H_{0} \cdot B_{0}\right) d V}
$$


where $E_{0}$ and $D_{0}$ are the electric field and electric field displacement in the empty resonator, $E_{1}$ and $D_{1}$ are the perturbed fields upon insertion of the sample. $B_{0}$ and $H_{0}$ are the magnetic flux intensity and magnetic field strength in the empty resonator, and $B_{1}$ and $H_{1}$ are perturbed fields in the presence of the sample. Because the electric and magnetic fields are decoupled in the resonator, the perturbation equation allows easy access to the dielectric and magnetic properties of the material. As an example, for dielectric measurements, $\boldsymbol{H}_{1}, \boldsymbol{H}_{0}, \boldsymbol{B}_{1}$, and $\boldsymbol{B}_{0}$ are 0 at the sample location and for the magnetic measurements $E_{1}, E_{0}, D_{0}$, and $D_{1}$ are 0 at the sample location. Using Waldron's notation [1], the complex frequency shift is related to the dielectric material parameters by

$$
\frac{\delta \omega}{\omega}+\frac{\mathrm{j}}{2}\left[\frac{1}{Q_{1}}-\frac{1}{Q_{0}}\right]=-A\left(\epsilon^{*}-1\right) \frac{2 y l_{1}}{l_{0}},
$$

where $\epsilon^{*}=\epsilon^{\prime}-\mathrm{j} \epsilon^{\prime \prime}, l_{1}$ is the half length of the sample, $y$ is the half thickness of the sample, $l_{0}$ is the length of the resonator, and $A$ is given by

$$
A=\frac{\pi \alpha}{2(\alpha+\beta) \mathrm{K}(1 / \alpha)} \sqrt{\frac{\alpha^{2}-\beta^{2}}{\alpha^{2}-1}},
$$

where $\mathrm{K}(1 / \alpha)$ is the complete elliptic integral of the first kind with modulus $1 / \alpha$. The parameter $A$ is a geometrical factor closely related to the dimensions $t, b$, and $w$.

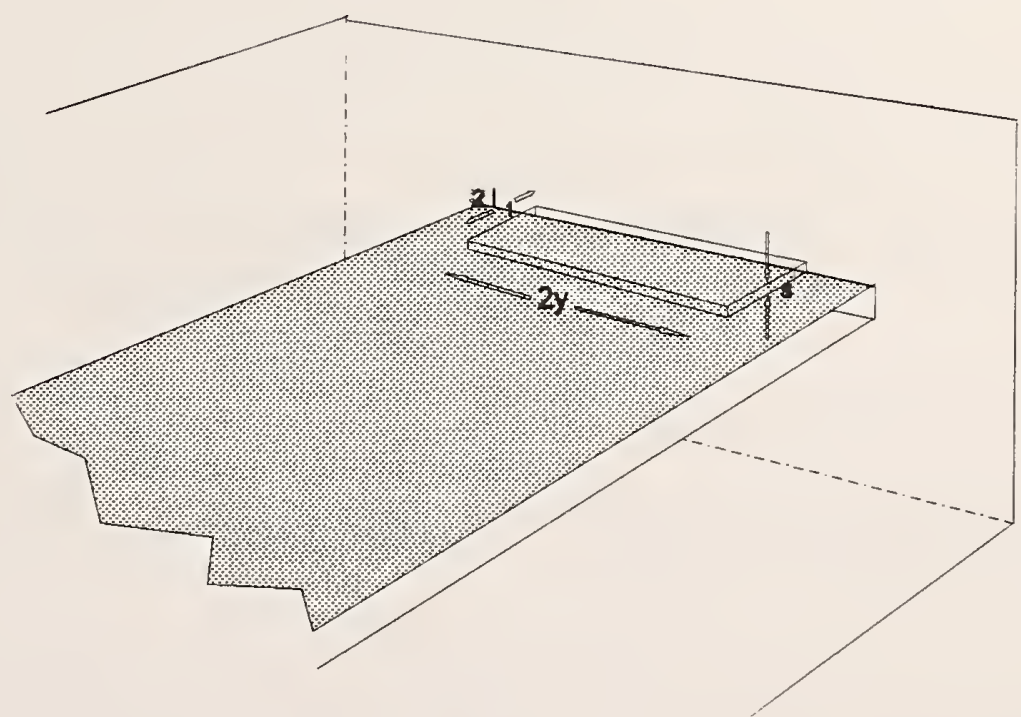

Case 3

Figure 3. Sample placement in maximum magnetic field for a magnetic property measurement in plane of the long axis of the sample and parallel to the center strip; see Ref. [1, Case 3]. 
Three formulas for measuring the anisotropy or the gyromagnetic factor of the material can be obtained by calculating the integral in eq. (5) for magnetic samples. Due to the geometry of the sample and its placement inside the cavity, as shown in Figure 3, only the following equation was used

$$
\frac{\delta \omega}{\omega}+\frac{\mathrm{j}}{2}\left[\frac{1}{Q_{1}}-\frac{1}{Q_{0}}\right]=-B\left(\mu^{*}-1\right) \frac{y l_{1} s}{b l_{0}(b-t)}
$$

where $\mu^{*}=\mu^{\prime}-\mathrm{j} \mu^{\prime \prime}, s$ is the thickness of the sample, $y$ is the width of the sample across the center conductor, and $l_{1}$ is the length of the sample measured from the end wall. The geometrical factor for magnetic measurements $B$ is given by

$$
B=\frac{A}{2}(1+\beta / \alpha) .
$$

The unloaded quality factor $Q_{0}$ of an empty resonator is obtained by integrating eq. (5) over the physical dimensions of the cavity:

$$
Q_{0}=\frac{\pi(b-t) \sqrt{120 \sigma}}{\sqrt{\lambda_{0}}\left[1+2(b-t) / l_{0}\right]},
$$

where $\sigma$ is the conductivity of the material, $\lambda_{0}$ is the wavelength in free space, $(b-t)$ is the air gap between ground plane and center conductor, and $l_{0}$ is the length of the cavity.

\subsection{Depolarization and Demagnetization}

A major cause of degradation of complex magnetic permeability measurements is demagnetization. This effect can be described by the equation

$$
\boldsymbol{H}_{\boldsymbol{t}}=\boldsymbol{H}_{\text {ext }}-N M,
$$

where $N$ is the demagnetization factor, $\boldsymbol{H}_{\text {ext }}$ is the applied microwave field in the resonator, $\boldsymbol{H}_{\mathrm{t}}$ is the observed magnetic field, and $M$ is the magnetization in the material. There are three ways to minimize the demagnetization effect: (a) use sample shapes where the demagnetization factor can 
be calculated accurately, (b) minimize the demagnetization factor by decreasing the cross-sectional area of the sample perpendicular to the field lines, or (c) use a combination of (a) and (b) by using long, thin samples so that the rectangular sample can be treated as an ellipsoid. Magnetization is homogeneous over the volume of spherical and ellipsoidal samples and can be calculated exactly [6, 13]. Shapes such as spheres or ellipsoids are not practical and cannot be measured accurately using the perturbation theory or mapping function. The perturbation theory used to calculate the permeability and the mapping function used to derive the final formulae dictate using only rectangular samples. In rectangular samples, the magnetization varies from point to point within the material. This can be explained by considering the rectangular sample to be made of imaginary layers. The outer layers screen the induced magnetic moment developed in the inner layers so that the magnetization close to the end of the sample is maximum and in the middle of the sample, minimum. If the sample is placed in the position shown in Figure 3, the sample must lie flush against the center conductor and end wall of the cavity to obtain accurate measurements. Placing the sample in the positions for cases 1 and 2 shown in Figures 4 and 5, flush against the center conductor and perpendicular to the ground plane, allows us to use cylindrical samples. The demagnetization factor is calculated using formulas developed for infinitely long cylinders [10]. For this reason we used long, narrow, rectangular samples to minimize the demagnetization factor and the screening effect. The samples were approximated as prolate ellipsoids with aspect ratio from 10 to 40 . Rectangular samples have three axes denoted here as $a, b$, and $c$, where $a$ is along the $\mathrm{x}$-axis, $b$ is along the $\mathrm{y}$-axis and $c$ is along the z-axis.

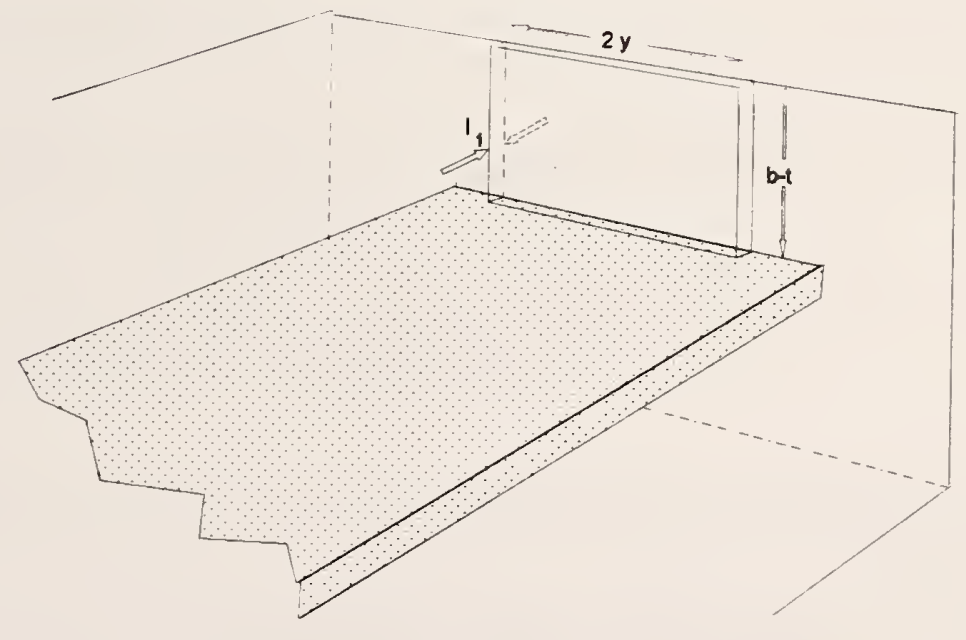

Figure 4. Sample placement for magnetic property measurement in plane of the long axis of the sample and normal to the center strip; see Ref.[1, case 1]. 


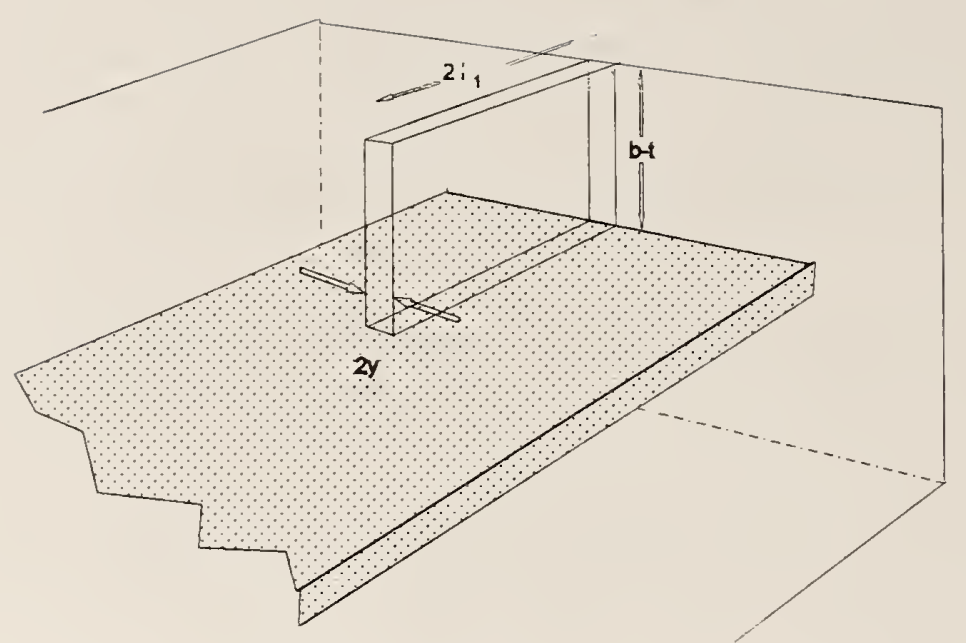

Figure 5. Sample placement for magnetic property measaurement in plane of the short axis and normal to the center strip; see [1, case 2].

If the sample is placed so that the magnetic field lines are along axis $c$, the demagnetization factor is [14]:

$$
D_{c}=\frac{a b c}{2} \int_{0}^{\infty} \frac{\mathrm{d} \lambda}{\left(c^{2}+\lambda\right) \mathrm{R}(\lambda)}
$$

where $a, b$, and $c$ are the dimensions of the rectangular sample, $D_{c}$ is the normalized demagnetization factor along axis $c$, and

$$
\mathrm{R}(\lambda)=\sqrt{\left(a^{2}+\lambda\right)\left(b^{2}+\lambda\right)\left(c^{2}+\lambda\right)}
$$

is a geometrical function. From symmetry considerations, similar formulas for $D_{a}$ and $D_{b}$ can be derived, where the relation between $D_{a}, D_{b}$, and $D_{c}$ is

$$
D_{a}+D_{b}+D_{c}=1 \text {. }
$$


In papers by Stoner [10] and Osborn [9], analytic formulas containing complete elliptic integrals are used for infinitely long rectangular samples. Browning also used this approach for his stripline resonator [11]. In our formulas, we used a well-known mathematical package to perform the integrations. Typical values for the demagnetization factor along $D_{c}$ range from 0.012 to 0.046 . This leads to large values of $D_{a}$ and $D_{b}$. Although no magnetic field was present along axes $a$ and $b, D_{a}$ and $D_{b}$ were calculated to check the accuracy of the numerical integrations using eq. (14).

To calculate the complex intrinsic magnetic properties $\mu_{\mathrm{i}}$ from the complex measurement results $\mu_{\mathrm{m}}$, we used the relation given by Musal [8],

$$
\left(\mu_{\mathrm{i}}-1\right)=\frac{\left(\mu_{\mathrm{m}}-1\right)}{1-D_{c}\left(\mu_{\mathrm{m}}-1\right)}
$$

This formula shows that the difference between $\mu_{\mathrm{m}}$ and $\mu_{\mathrm{i}}$ increases with increasing $\mu_{\mathrm{m}}$ and $\mu_{\mathrm{i}}$; it is zero if $D_{c}=0$ or $\mu_{\mathrm{i}}=\mu_{\mathrm{m}}=1$. For large values of $\mu_{\mathrm{i}}, \mu_{\mathrm{m}}$ approaches the limiting value of $\left(1+D_{c}\right) / D_{c}$. For example, if $D_{c}=0.02$, the limiting measurable value for $\mu_{\mathrm{m}}$ will be 51 , even if the intrinsic value is much higher.

\section{EXPERIMENTAL METHODOLGY}

The mechanical design of a stripline resonator has a significant effect on measurement accuracy. As a general rule of thumb, uniform fields are obtained within the sample when the ground planes are close to the center conductor. However, this leads to a small cavity volume resulting in a low quality factor. On the other hand, a high quality factor is desired in order to achieve high accuracy for lowloss materials. Since these criteria work in opposition, a compromise must be made as to cavity volume and quality factor. This section illustrates the procedure for determining the dimensions of a resonator. An example resonator will first be designed showing the necessary steps. Following this the dimensions we chose for our resonator will be presented.

\subsection{Cavity Design}

The first thing to consider when designing a stripline resonator is the lowest frequency at which measurements are to be made. Typically the length of the resonator is chosen to be one-half wavelength at the fundamental frequency. Cavity dimensions are chosen so that the field uniformity error is less than some chosen value. This error in the measurement is inherent to the design of the resonator and cannot be removed by changing the shape of the cavity or the material it is constructed with. For illustration, an upper bound of 0.1 percent error is arbitrarily chosen as shown in Figures 6 and 7. The next parameters chosen from the graphs are $\alpha$ and $\beta$ which determine how parallel the fields will be in the center of the cavity. If an angle of $89^{\circ}$ is chosen for $\beta$, and a value of 1.0002 for 


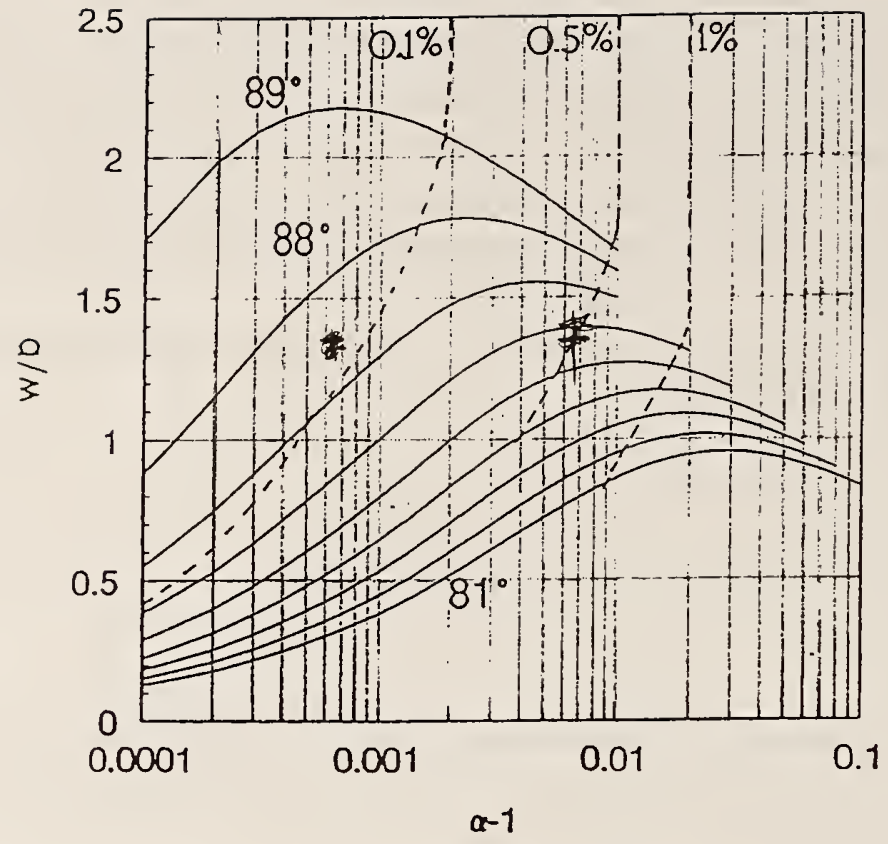

Figure 6. Ratio of stripline width-to-separation versus $\alpha$-1 for various values of the parameter $\sin ^{-1} \beta$; the locii curves of constant nonuniformity error are shown dashed.

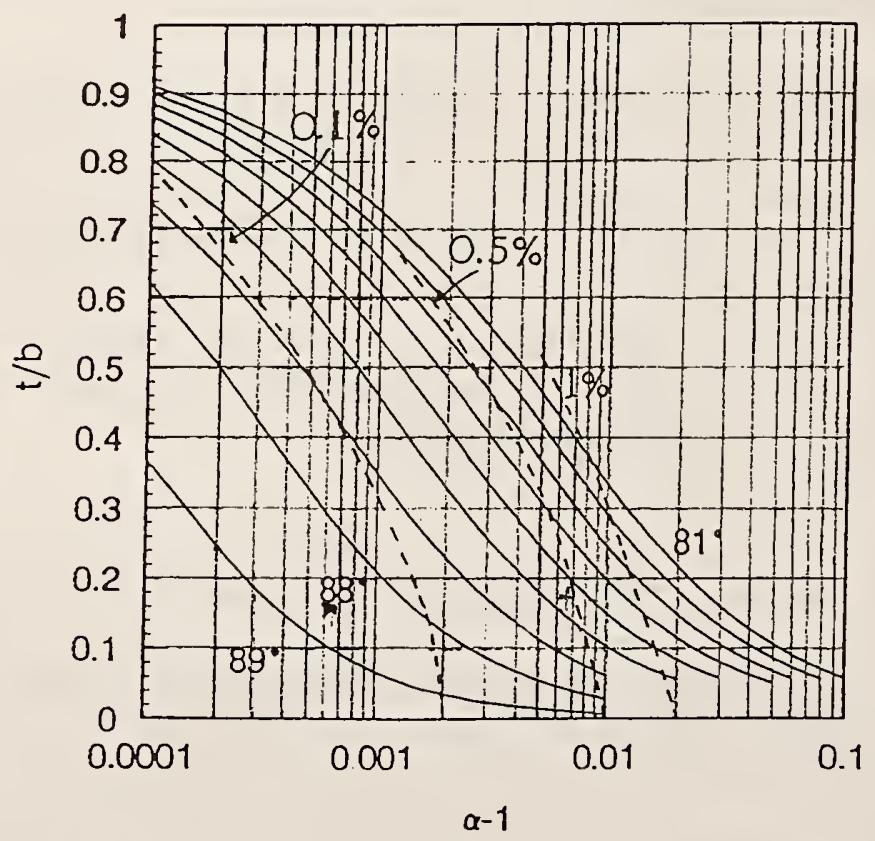

Figure 7. Ratio of stripline center plate thickness-to-separation versus $\alpha-1$ for various values of the parameter $\sin ^{-1} \beta$; locii curves of constant nonuniformity error are shown dashed. 
$\alpha$, and the separation between the ground planes is $2 b$ then a determination of the width and thickness of the center conductor can be obtained. For example, if $2 b=5.08 \mathrm{~cm}$ then $b=2.54 \mathrm{~cm}$ and from all other parameters chosen above it can be determined, from Figures 6 and 7, that $w / b=2$ and $t / b$ $=0.24$. Therefore, the width $2 w$ of the center conductor should be $10.16 \mathrm{~cm}$ and the thickness $2 t$ of the center conductor should be $1.22 \mathrm{~cm}$. These choices will ensure that the physical dimensions of the cavity will not contribute more than 0.1 percent error to the measurement.

The resonator we designed has a fundamental frequency of $150 \mathrm{MHz}$, for a TEM mode, with harmonics every $150 \mathrm{MHz}$. The error in design was chosen to be 0.01 percent. From the design equations given in Section 2.1, $\alpha$ was chosen to be 1.0067 and $\beta$ was chosen to be 0.9971 (sin $85.6^{\circ}$ ). The ratio $w / b$ of the width to the ground plane separation was 1.3333 and the ratio $t / b$ of the thickness to the ground plane separation was 0.16667 , for a ground plane separation $b$ of $7.62 \mathrm{~cm}$. Therefore, the total width of our center conductor is $10.16 \mathrm{~cm}$, and the total thickness of the center conductor is $1.27 \mathrm{~cm}$. The ground planes are $0.635 \mathrm{~cm}$ thick. The parameters $\alpha$ and $\beta$ were also chosen so that the resonator would have an optimal quality factor for the chosen error tolerance in the design.

\subsection{Quality factor}

The quality factor is determined not only by the volume of the cavity and the conductivity of the cavity walls, as given in eq. (10), but also by the radiation losses in the system. The theoretical and measured unloaded quality factors of this resonator are shown in Figure 8. The effects of conductor

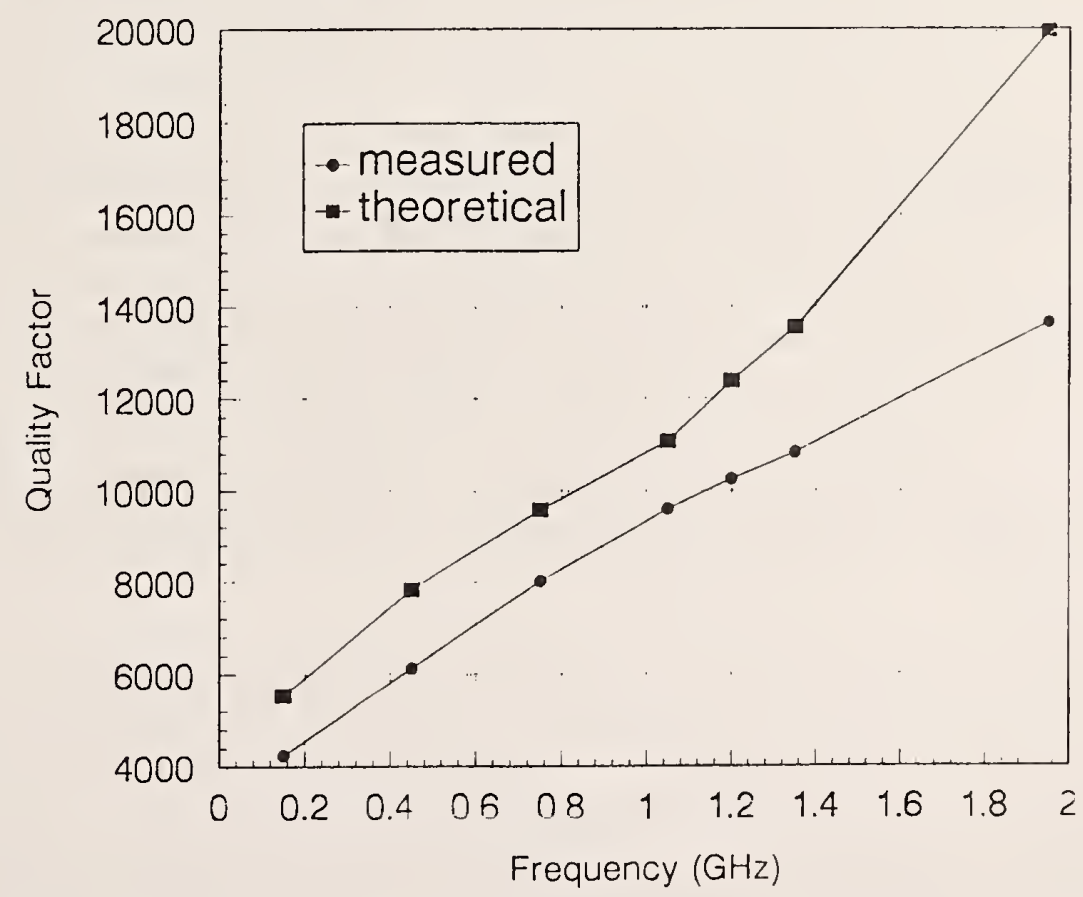

Figure 8. Theoretical and measured unloaded quality factors for the NIST resonator. 
and radiation losses (see below) have been accounted for in the theoretical computation. Losses due to surface currents found in the joints of the resonator, the loading of the system due to the coupling mechanism, and radiation leakage from the open sides of the resonator can be minimized. To minimize joint losses, a conductive paste was used to provide conductive contact between the joints. Loop coupling was chosen to excite the fields instead of the electric field coupling used in Waldron and Maxwell's [1,2] resonator. The main concern here was that electric field coupling is accomplished by moving the probe along a slot in the ground plane which can lead to radiative losses. However, loop coupling will always excite the magnetic field at the end wall of the cavity. These loops are shown in Figure 1. Small loops were made so that the insertion loss into the system is minimal; therefore, the loaded quality factor of the cavity (with loops) is close to the unloaded quality factor of the cavity (without loops) and a large correction does not have to be made.

To minimize radiation leakage from the sides, the minimum width of the ground planes can be determined according to the formula derived by Waldron [4],

$$
C=\frac{b}{\pi} \ln \left[y^{2} \mathrm{~K}^{2}(1 / \alpha) \frac{\sqrt{1-\frac{1}{\alpha^{2}}}}{2 \sqrt{1-\beta^{2}}}\right],
$$

until only a certain percentage of radiation leaks beyond some point $C$, shown in Figure 2. Collin [17] demonstrates that the ratio of the width of the ground plane to the width of the center conductor should be approximately 3 to keep radiation losses to a minimum. The other alternative is to install side walls with access doors on the open sides of the resonator, creating a structure very similar to a resonant TEM cell. The closed resonator gave more accurate results for dielectric loss measurements because radiation losses were eliminated. However, the side walls cause higher-order modes to propagate at lower frequencies than is the case for the open-sided structure [16]. The resonance spectra for the structure both with and without side walls are shown in Figure 9. The higher-order mode resonances can interfere with the resonances of interest, so one must be careful to ensure that the resonance is clean before measuring the quality factor. Radiation loss can also occur if the ground planes are not parallel. To help maintain parallelism, the stripline was suspended and the ground planes were supported using a metal frame. Radiation leakage increased upon insertion of the dielectric sample. To minimize this leakage, nearly identical samples were placed symmetrically on either side of the center conductor. 


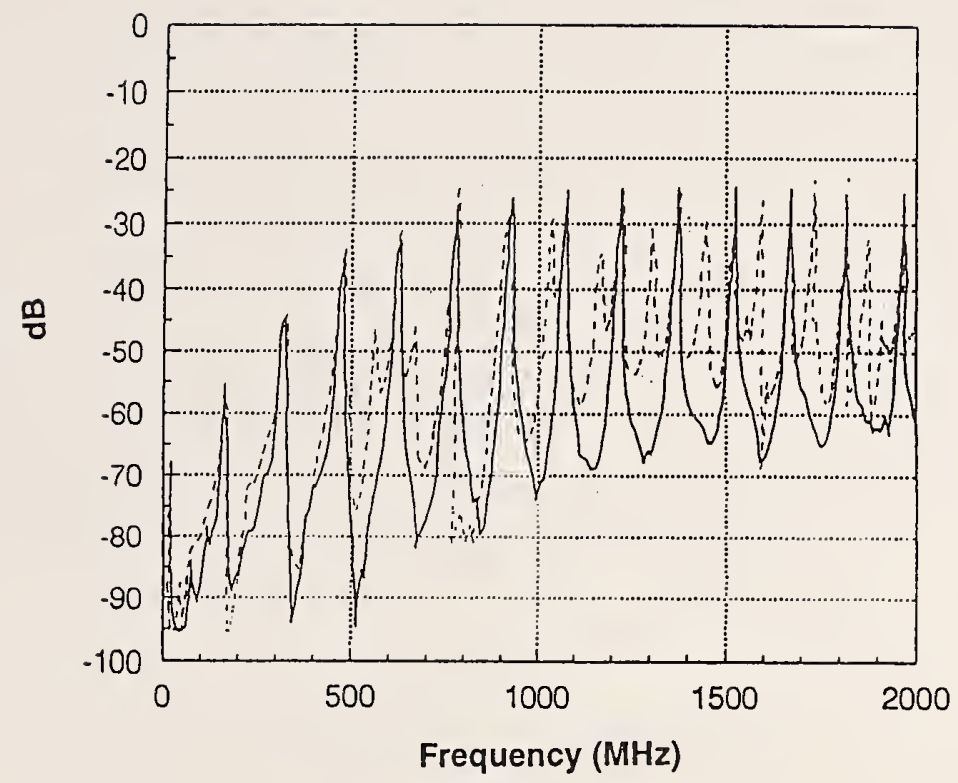

Figure 9. Resonance spectra of the stripline resonator for case without side walls (lines) and with side walls (dashed lines).

\section{MEASUREMENT RESULTS}

The main purpose of the stripline resonator is to measure the properties of permeable materials. Therefore, this section concentrates primarily on measurements of magnetic materials. Cross-linked polystyrene, a well-characterized dielectric material, was measured and used as a reference standard to gauge the accuracy of the resonator. A comparison was made to measurements taken with a $7 \mathrm{~mm}$ coaxial transmission line as well as to the accepted value of 2.55 for the dielectric constant and 0.00038 for the loss tangent at $100 \mathrm{MHz}$ [18]. Values for a low-loss dielectric material are not expected to vary much with frequency. These results are shown in Figure 10. The measurement of the loss tangent is inaccurate and not shown. Because the loss tangent is related to the measured quality factor, when radiation losses are large, measurement of loss tangent will be inaccurate. For this reason, the resonator is symmetrically loaded as discussed in the previous section.

The first measurements of magnetic properties were taken on a relatively low-loss magnetic material, followed by measurements on a sample with high values of both the real and imaginary components. Table 1 summarizes the measured samples, their various dimensions and the demagnetization factor calculated for each one along the axis parallel to the magnetic field. $D_{c}$ is the demagnetization factor in the direction of the applied field, $t_{\mathrm{s}}$ is the thickness of the sample, $w_{\mathrm{s}}$ is the width of the sample and $l_{\mathrm{s}}$ is the length of the sample. According to Waldron's formula (8), $t_{\mathrm{s}}=s$, 


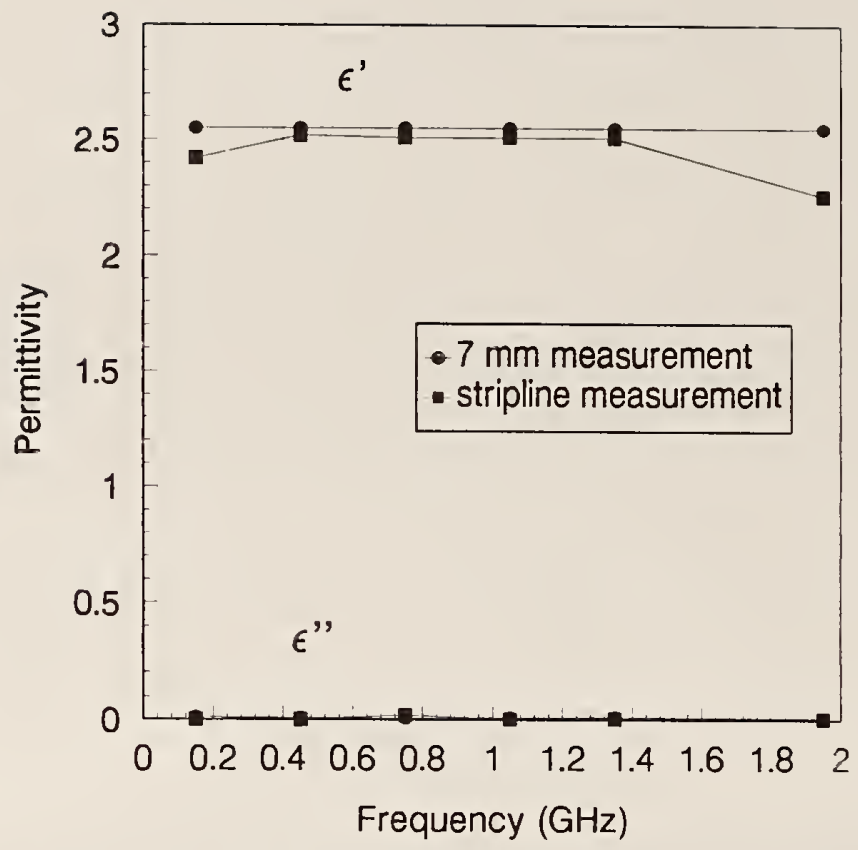

Figure 10. Measurement results for cross-linked polystyrene, compared with data from $7 \mathrm{~mm}$ coaxial transmission line measurements.

Table 1. Physical parameters of magnetic samples.

\begin{tabular}{ccccc}
\hline \hline material & $t_{\mathrm{s}}$ & $w_{\mathrm{s}}$ & $l_{\mathrm{s}}$ & $D_{c}$ \\
\hline $\begin{array}{c}\text { Loaded polymer } \\
\text { sample G }\end{array}$ & $3.19 \mathrm{~mm}$ & $9.99 \mathrm{~mm}$ & $70.1 \mathrm{~mm}$ & 0.038 \\
$\begin{array}{c}\text { Nickel-zinc ferrite } \\
\text { sample A }\end{array}$ & $3.19 \mathrm{~mm}$ & $3.96 \mathrm{~mm}$ & $31.5 \mathrm{~mm}$ & 0.067 \\
$\begin{array}{c}\text { Nickel-zinc ferrite } \\
\text { sample D }\end{array}$ & $1.03 \mathrm{~mm}$ & $4.00 \mathrm{~mm}$ & $42.5 \mathrm{~mm}$ & 0.017 \\
\hline
\end{tabular}

$w_{\mathrm{s}}=l_{1}$, and $l_{\mathrm{s}}=y$, as shown in Figure 3. Table 1 shows that all samples have $10<l_{\mathrm{s}} t_{\mathrm{s}}<42$. Samples were placed flush against the end wall and center conductor of the cavity as shown in Figure. 1 . The measured and corrected results for the loaded polymer $\mathrm{G}$ are shown in Figure 11 and compared to a measurement in a $7 \mathrm{~mm}$ coaxial transmission line. As one can see, the effects on the loaded polymer due to demagnetization are quite small. These effects are small because (a) the sample is long and 
thin, $l_{\mathrm{s}} /_{\mathrm{s}}=21.97$ and (b) $\mu^{\prime} \approx 4$, which is a lower limit in the demagnetization effect as discussed by Maxwell [2] and Musal [8]. Figure 11 shows that $\mu^{\prime}$ varies from approximately 3.9 to 4.5 over the frequency range from $150 \mathrm{MHz}$ to $2 \mathrm{GHz}$. The imaginary component $\mu^{\prime \prime}$ increases from 0 at 150 $\mathrm{MHz}$ to approximately 3 at $1.95 \mathrm{GHz}$ for the stripline resonator. The measurements for the $7 \mathrm{~mm}$ coaxial transmission line are similar, but $\mu^{\prime}$ decreases more rapidly and $\mu^{\prime \prime}$ does not increase as rapidly as in the stripline resonator. The material aspect ratios were machined to have values $10<l_{1} / t_{\mathrm{s}}<40$. The ratio of length to width of the samples had values $8<l_{1} / w_{\mathrm{s}}<10$ and each of these was chosen to decrease the demagnetization effect. Although steps were taken to reduce the demagnetization effect, this effect remains large. Figures 12 and 13 show the measured and corrected results for the nickel-zinc ferrite samples A and D. In Figures 12 to 14, the open symbols show the measured results before the demagnetization correction and the filled symbols show the results for the real and imaginary parts of permeability after correction. The samples A and D were machined from the same block of material, so the permeability should be approximately the same.

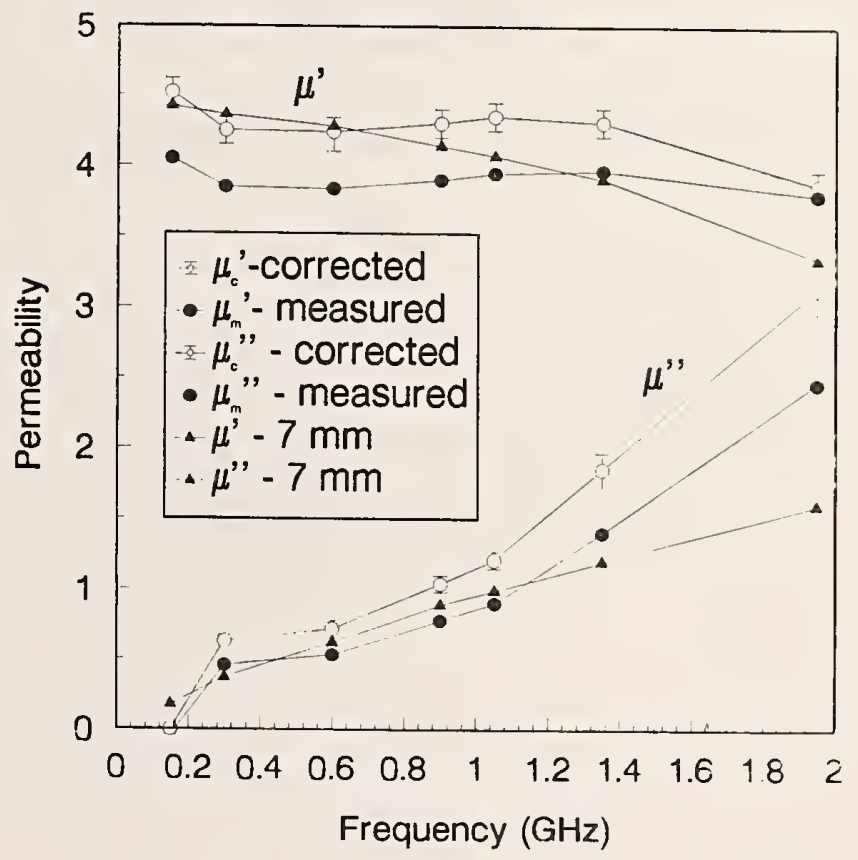

Figure 11. Measured and corrected results for the permeability of a low-loss loaded polymer compared with data from $7 \mathrm{~mm}$ coaxial transmission line measurements.

Figs. 12 and 13 show that the correction for demagnetization is very large at low frequencies and decreases approximately exponentially toward the higher frequencies owing to the behavior of the intrinsic properties $\mu_{\mathrm{i}}^{\prime}$ and $\mu_{\mathrm{i}}^{\prime \prime}$. The corrected permeability $\mu_{\mathrm{c}}^{\prime}$ changes from $20 \pm 1.5$ at $150 \mathrm{MHz}$ to 


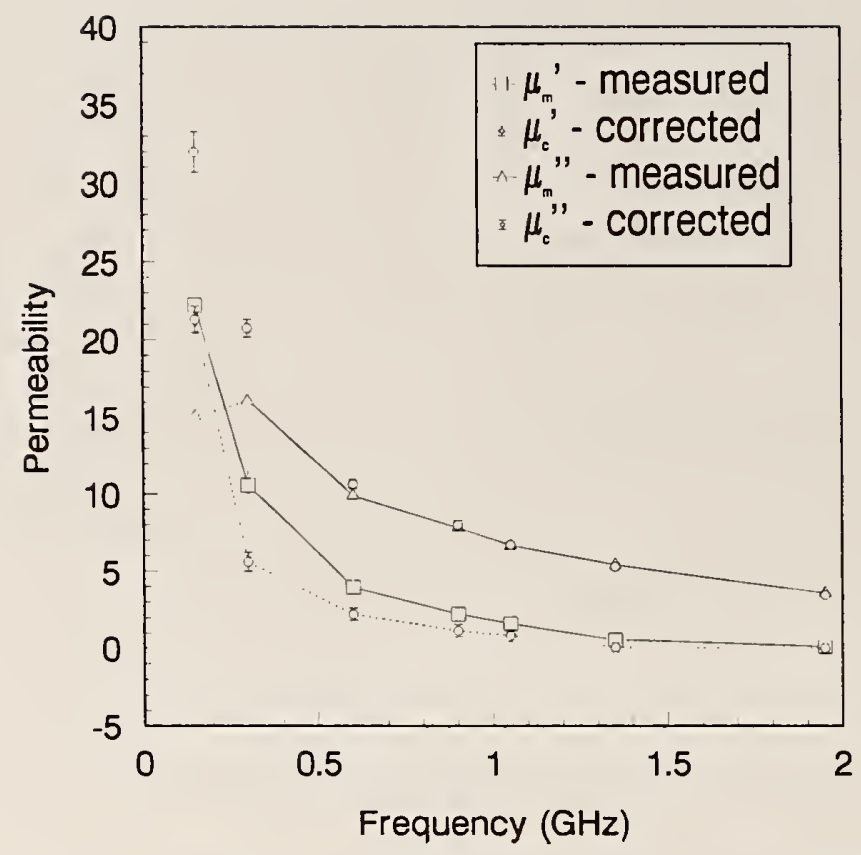

Figure 12. Measured and corrected permeability values for a nickel-zinc ferrite, sample $A$

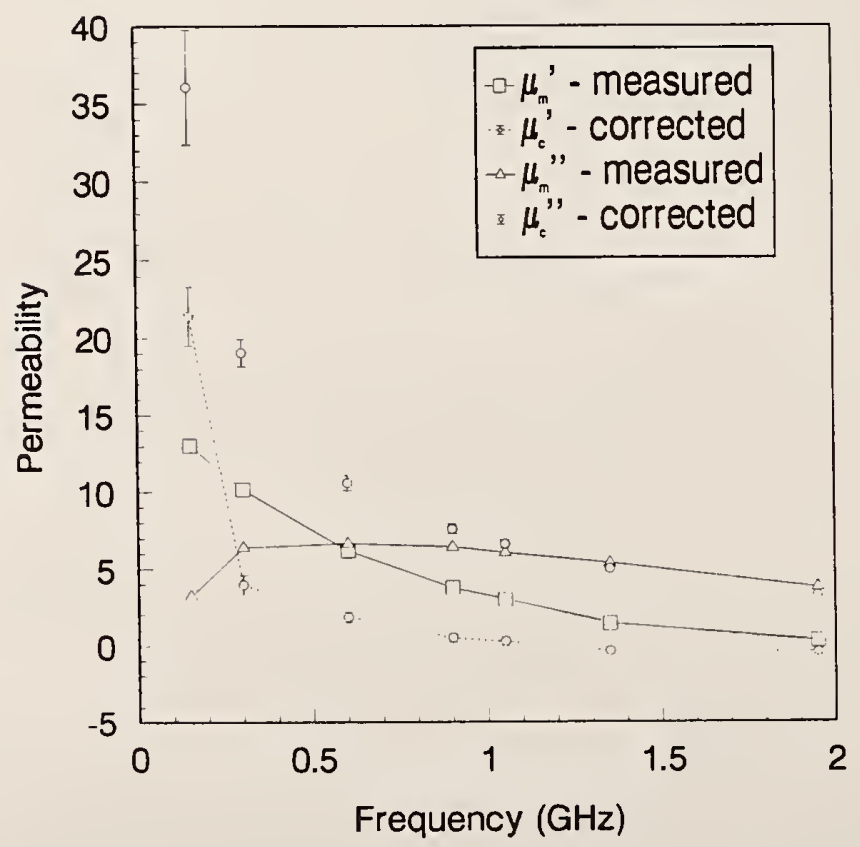

Figure 13. Measured and corrected permeability values for a nickel-zinc ferrite, sample D. 
approximately $0 \pm 0.5$ at $1.95 \mathrm{GHz}$. There is a change in $\mu_{\mathrm{c}}{ }^{\prime \prime}$ from $35 \pm 3$ at $150 \mathrm{MHz}$ to approximately $4 \pm 0.2$ at $1.95 \mathrm{GHz}$. Here and throughout, the quoted uncertainties are standard uncertainties, i.e., one standard deviation estimates [19]. In both figures the measured results are much lower than the expected values for the intrinsic magnetic properties. Most of the effect of demagnetization is seen in the imaginary part of the permeability, where the values actually decrease at the lower frequencies instead of increasing as the corrected value shows. From Table 1, we see that sample $\mathrm{D}$ is narrower and longer than sample $\mathrm{A}$, in order to more closely approximate the sample $\mathrm{D}$, to an ellipsoid; hence the correction is not nearly as large for sample D, as it is for sample A. These results show that longer, thinner samples require less correction.

Figure 14 summarizes the corrected values for samples $A$ and $D$ including a comparison to a $7 \mathrm{~mm}$ coaxial measurement. The two stripline samples show the same trend within the error bounds and are very close to measurements made in the $7 \mathrm{~mm}$ coaxial transmission line which used a sample machined from a different lot but assumed to have similar properties. Both techniques show the same tendencies for this magnetic material which have very high magnetic permeability at low frequencies. The discrepancies can be a result of the variability between lots of material. As we have shown, demagnetization has a large effect on the measurement results so care must be taken in the preparation of the samples.

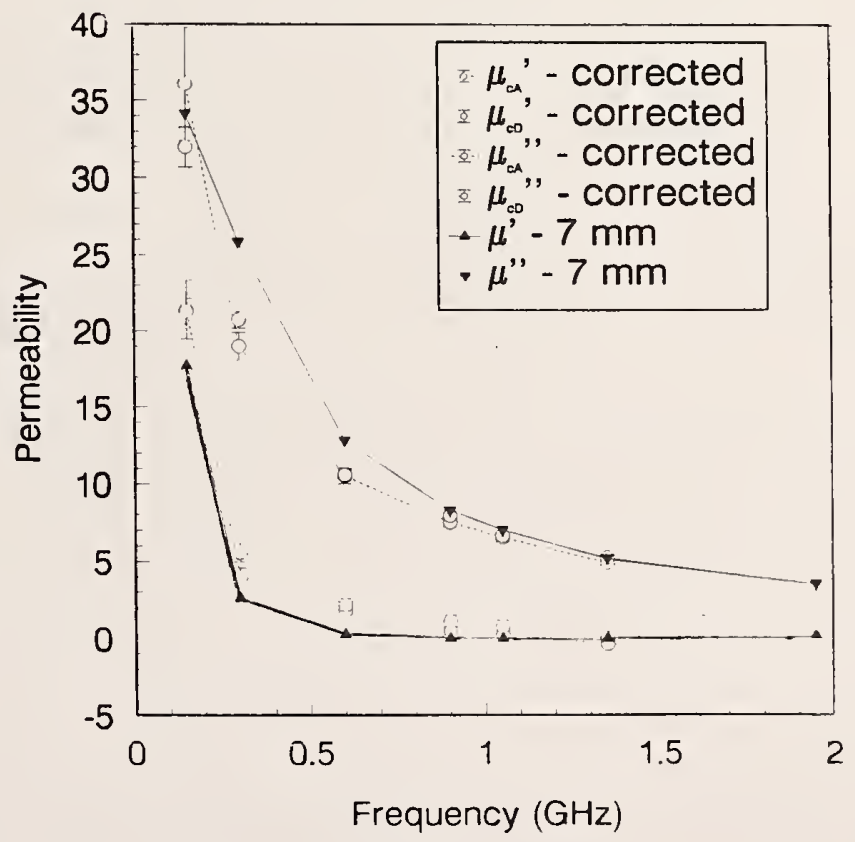

Figure 14. Corrected values for nickel-zinc ferrite samples A and D compared with data from $7 \mathrm{~mm}$ coaxial transmission line measurements. 


\section{ERROR ANALYSIS}

The permeability and permittivity measurement uncertainties are divided into two parts: (a) uncertainties associated with systematic effects due to the design of the resonator resulting in nonuniform fields within the sample, and (b) uncertainties associated with random effects due to random changes in the procedure.

The systematic errors were investigated by analyzing the design of a prototype resonator built previously. The dimensions of this resonator were $t / b=0.083$ and $w / b=0.67$, so $\alpha=1.018$ and $\beta$ $=84^{\circ}$. When cross-linked polystyrene was measured in this cavity, $\epsilon_{\mathrm{r}}^{\prime}=2.30$ was obtained with an experimental standard deviation of \pm 0.1 , instead of the accepted value of 2.55 . To ensure that the error was not a result of a frequency shift outside the assumptions of the perturbation model, the dimensions of the sample were decreased with no noticeable change in the results. It appears that the error was due to nonuniform electric field lines within the sample due to design errors in the width and thickness of the center conductor. To improve these results, the thickness of the center conductor was increased to $12.7 \mathrm{~mm}$ from $6.4 \mathrm{~mm}$, and the width of the center conductor was increased to $10.16 \mathrm{~cm}$ from $5.08 \mathrm{~cm}$. The ratio of thickness to separation was thus $t / b=0.167$, and the ratio of width to separation was thus $w / b=1.33$. The accuracy of the measurement for cross-linked polystyrene improved considerably after redesign; specifically, $\epsilon_{\mathrm{r}}^{\prime}=2.50 \pm 0.02$ (Figure 10). Choosing a nonoptimal or inappropriate $\alpha$ and $\beta$ can have a large effect on the results.

Uncertainties associated with random effects in measurements of $\delta \omega / \omega$ and $1 / Q$ arose due to the mechanical instability of our copper resonator. A slight displacement of the sample from the center of the cavity where the electric field is uniform will result in another source of uncertainty. Uncertainty components arisng from measurements of $\mu_{\mathrm{m}}{ }^{*}$ and $\mu_{\mathrm{i}}{ }^{*}$ were Monte Carlo simulated. A randomly generated distribution of 100 measurements was calculated. The estimated standard deviation in the demagnetization factor $D_{c}$ was \pm 0.025 , the estimated random uncertainties for the frequency change $\delta \omega / \omega$ and inverse quality factor $Q$ were $1.2 \times 10^{-5}$ and 0.036 , respectively. The above estimated uncertainties were inserted into (15) and (18), using the measured frequency shift and change in quality factor. The mean value and one standard deviation uncertainty for $\mu_{\mathrm{m}}{ }^{\prime}, \mu_{\mathrm{m}}{ }^{\prime \prime}$, $\mu_{\mathrm{i}}^{\prime}, \mu_{\mathrm{i}}^{\prime \prime}$ were calculated for every measured frequency and led to the error bars shown in Figures 12 through 14.

\section{SUMMARY AND CONCLUSIONS}

This report has presented the stripline resonator method, including the theoretical background, critical mechanical design of the cavity, and experimental results, for magnetic materials.

Conformal mapping was used to describe the electromagnetic fields in the resonator, mainly the fields in the location of the sample. Uniform fields must be achieved in the sample in order to properly use 
the formulas for $\epsilon^{*}$ and $\mu^{*}$. Perturbation theory was used to derive the formulas for calculation of the dielectric and magnetic properties from changes in the resonant frequencies and quality factors of the cavity. Both low- and high-loss materials were measured in this work.

Conformal mapping as a mathematical method to describe the electromagnetic fields in the entire volume of the resonator is complicated. In the future, numerical techniques such as finite difference methods, finite-element analysis, and Green's function analysis can be implemented to describe the behavior of the fields inside the cavity with and without a sample. With these techniques we hope to determine the resonant frequencies and quality factors with and without a sample, so that perturbation conditions are not required. By comparing experimental results to the numerical results, we can use a numerical iteration procedure to obtain the permittivity and permeability of a sample.

We must understand the basic assumptions and the limitations of the theory in order to build the appropriate resonator. In the mechanical design section, the inherent errors in measuring the dielectric properties were related to the resonator dimensions $t, w$, and $b$. Choosing $\alpha$ and $\beta$ very close to 1 can eliminate systematic uncertainties of \pm 5 percent or more in the dielectric constant due to nonuniformity of the electric fields in the sample. Numerical examples using Figures 6 and 7 and experimental results from a prototype resonator were shown to demonstrate how results are affected. An interrelationship exists between a high quality factor of the resonator, its dimensions $\mathrm{t}, w$ and $b$, and the uniformity of the fields in the sample location. These properties tend to oppose each other, so compromises must be made in the design of the cavity. Figure 9 shows the quality factor of our resonator. Measurements can be taken using removable metal doors to close the cavity in order to minimize the radiation losses. Better dielectric results were achieved in this way. One must look for higher-order modes that can appear as a result of closing the resonator, as shown in Figure 8. For magnetic measurements, when the sample is located at the end wall, there is no need to close the cavity.

In Sections 2 and 4 we showed three measurements (Cases 1,2, and 3) that can be performed on thin rectangular plate samples in order to determine the elements of the permeability tensor of a ferrite. Case 3 is used here so that there is no inherent error in the cavity design because the sample does not occupy the full height of the cavity. The results obtained for magnetic materials depend strongly on the sample dimensions. Samples from the same bulk but with different dimensions exhibit totally different results for both $\mu^{\prime}$ and $\mu^{\prime \prime}$, especially at low frequencies where $\mu^{\prime}$ and $\mu^{\prime \prime}$ are high. This results from the demagnetization effect and is described in detail in the paper. Long and thin rectangular samples were used in order to implement the ellipsoid model. This model states that if the ratios $l_{\mathrm{s}} / t_{\mathrm{s}}$ and $l_{\mathrm{s}} / w_{\mathrm{s}}$ are large enough, we can assume that the magnetic field inside the sample is very close to the magnetic field in an ellipsoid, which can be calculated by using analytical formulas. This model works well, and the corrected results for $\mu_{\mathrm{m}}{ }^{\prime}$ and $\mu_{\mathrm{m}}{ }^{\prime \prime}$ in samples with different dimensions lie within the experimental uncertainty. The measurements taken in the $7 \mathrm{~mm}$ coaxial transmission line show good agreement with the stripline resonator measurements. These results are shown in Figure 12.

The sources of uncertainties associated with random effects are mainly radiation losses in the 
dielectric measurements, and errors in placement of the samples in either permittivity or permeability measurements. The uncertainty bounds for the loaded polymer, which has low permeability, were small and did not change much with frequency, especially when $f \gg>150 \mathrm{MHz}$. The demagnetization correction is small, as is expected for low permeability materials. The uncertainties for nickel-zinc ferrite, which has high permeability, are shown in Figures 12 to 14 . The combined standard uncertainties are greatest at $150 \mathrm{MHz}$ and decrease at the higher frequencies. At $150 \mathrm{MHz}$, generally the nickel-zinc ferrites have relative errors of \pm 4 percent for $\mu_{\mathrm{i}}^{\prime}$ and \pm 10 percent for $\mu_{\mathrm{i}}^{\prime \prime}$. At the higher frequencies, the relative errors for $\mu_{i}^{\prime}$ remain unchanged from \pm 4 percent; however, the relative errors for $\mu_{\mathrm{i}}^{\prime \prime}$ increase dramatically to $\pm(20$ percent to 60 percent $)$ as $\mu_{\mathrm{i}}$ " approaches 0 above $1 \mathrm{GHz}$. Although the demagnetization factors for these samples were low, the demagnetization was large, especially at low frequencies, where the permeability is high. But the major contributions to the uncertainties were dimensional uncertainties associated with $\mu_{\mathrm{m}}{ }^{\prime}$ and $\mu_{\mathrm{m}}{ }^{\prime \prime}$, and only minor contributions from uncertainties in calculations of the demagnetization factors.

It is important to emphasize that the above uncertainties for $\mu^{\prime}$ and $\mu^{\prime \prime}$ are a result of our uncertainty analysis of the redesign of the resonator and the correction for demagnetization. Without the redesign, uncertainties of approximately 10 percent for $\mu^{\prime}$ and 15 percent to 20 percent for $\mu^{\prime \prime}$ would have resulted at $150 \mathrm{MHz}$. Similarly, uncertainties for $\epsilon$ have been reduced from 5 percent to 1 percent for cross-linked polystyrene.

We thank Douglas Gallagher and Jim Boyd of the NIST-Boulder Instrument Shops for their suggestions and help with the fabrication of our resonator fixture.

\section{REFERENCES}

[1] Waldron, R.A. Theory of a strip-line cavity for measurement of dielectric constants and gyromagnetic-resonance line-widths, IEEE Trans. Microwave Theory Tech., MTT-12: 123131; January 1964.

[2] Maxwell, S. A stripline cavity resonator for measurement of ferrites, Microwave J., 9: 99102; 1966.

[3] Waldron, R.A. Theory of the strip-line cavity resonator, Marconi Rev., First Quarter, 27: 3042; 1964. 
[4] Maxwell, S.P. Strip-line cavity resonator for measurement of magnetic and dielectric properties of ferrites at low microwave frequencies, Marconi Rev., First Quarter, 27: 22-29; 1964.

[5] Fox, J.; Sucher, M. Handbook of microwave measurements, Polytechnic Institute of Brooklyn, Microwave Research Institute, New York: 777-778; 1963.

[6] Stratton, J.A. Electromagnetic theory: McGraw-Hill Inc., London; 1941.

[7] Waldron, R.A.; Maxwell, S.P. Note on the measurement of material properties by the stripline cavity, IEEE Trans. Microwave Theory Tech., MTT-13: 711; 1965.

[8] Musal, H.M. Demagnetization effect in strip-line cavity measurements, IEEE Trans. Magn., 28: 3129-3131; 1992.

[9] Osborn, J.A. Demagnetizing Factors of the General Ellipsoid, Phys. Rev., 67:351-357; 1945.

[10] Stoner, E.C. The Demagnetizing Factors for Ellipsoids, Phil. Mag. Ser. 7, 36 (263): 803-821; 1945.

[11] Browning, S.L.; Westbrook, E.P. Stripline resonator design for material characterization, Proceedings of the AMTA Workshop, Chicago, IL; July 1992.

[12] Waldron, R.A. Perturbation theory of resonant cavities, IEE Monograph, 373E: 272-294; 1960.

[13] Jackson, J.D. Classical electrodynamics: John Wiley \& Sons, Inc., New York; 1962.

[14] Becker, R. Electromagnetic fields and interactions: Blaisdell Publishing Company; 1964.

[15] Waldron, R.A. Theory of guided electromagnetic waves: pp. 140-145; Van NostrandReinhold, London; 1969.

[16] Weil, C.M.; Gruner, L. High-order mode cutoff in rectangular striplines, IEEE Trans. Microwave Theory Tech. MTT-32: 638-641; 1984. 
[17] Collin, R.E. Field theory of guided waves, Second Edition: IEEE Press; 1991.

[18] Von Hippel, A.R. Dielectric materials and applications, p. 340: MIT Press, Cambridge, MA; 1954.

[19] Taylor, B.N.; Kuyatt, C.E. Guidelines for evaluating and expressing the uncertainty of NIST measurement results, Natl. Inst. Stand.Technol. Tech. Note 1297, 1994. 


\section{NIST Technical Publications}

\section{Periodical}

Journal of Research of the National Institute of Standards and Technology-Reports NIST research and development in those disciplines of the physical and engineering sciences in which the Institute is active. These include physics, chemistry, engineering, mathematics, and computer sciences. Papers cover a broad range of subjects, with major emphasis on measurement methodology and the basic technology underlying standardization. Also included from time to time are survey articles on topics closely related to the Institute's technical and scientific programs. Issued six times a year.

\section{Nonperiodicals}

Monographs-Major contributions to the technical literature on various subjects related to the Institute's scientific and technical activities.

Handbooks-Recommended codes of engineering and industrial practice (including safety codes) developed in cooperation with interested industries, professional organizations, and regulatory bodies.

Special Publications-Include proceedings of conferences sponsored by NIST, NIST annual reports, and other special publications appropriate to this grouping such as wall charts, pocket cards, and bibliographies.

Applied Mathematics Series-Mathematical tables, manuals, and studies of special interest to physicists, engineers, chemists, biologists, mathematicians, computer programmers, and others engaged in scientific and technical work.

National Standard Reference Data Series-Provides quantitative data on the physical and chemical properties of materials, compiled from the world's literature and critically evaluated. Developed under a worldwide program coordinated by NIST under the authority of the National Standard Data Act (Public Law 90-396). NOTE: The Journal of Physical and Chemical Reference Data (JPCRD) is published bimonthly for NIST by the American Chemical Society (ACS) and the American Institute of Physics (AIP). Subscriptions, reprints, and supplements are available from ACS, 1155 Sixteenth St., NW, Washington, DC 20056.

Building Science Series-Disseminates technical information developed at the Institute on building materials, components, systems, and whole structures. The series presents research results, test methods, and performance criteria related to the structural and environmental functions and the durability and safety characteristics of building elements and systems.

Technical Notes-Studies or reports which are complete in themselves but restrictive in their treatment of a subject. Analogous to monographs but not so comprehensive in scope or definitive in treatment of the subject area. Often serve as a vehicle for final reports of work performed at NIST under the sponsorship of other government agencies.

Voluntary Product Standards-Developed under procedures published by the Department of Commerce in Part 10, Title 15, of the Code of Federal Regulations. The standards establish nationally recognized requirements for products, and provide all concerned interests with a basis for common understanding of the characteristics of the products. NIST administers this program in support of the efforts of privatesector standardizing organizations.

Consumer Information Series-Practical information, based on NIST research and experience, covering areas of interest to the consumer. Easily understandable language and illustrations provide useful background knowledge for shopping in today's technological marketplace.

Order the above NIST publications from: Superintendent of Documents, Government Printing Office, Washington, DC 20402.

Order the following NIST publications-FIPS and NISTIRs-from the National Technical Information Service, Springfield, VA 22161.

Federal Information Processing Standards Publications (FIPS PUB)-Publications in this series collectively constitute the Federal Information Processing Standards Register. The Register serves as the official source of information in the Federal Government regarding standards issued by NIST pursuant to the Federal Property and Administrative Services Act of 1949 as amended, Public Law 89-306 (79 Stat. 1127), and as implemented by Executive Order 11717 (38 FR 12315, dated May 11, 1973) and Part 6 of Title 15 CFR (Code of Federal Regulations).

NIST Interagency Reports (NISTIR)-A special series of interim or final reports on work performed by NIST for outside sponsors (both government and non-government). In general, initial distribution is handled by the sponsor; public distribution is by the National Technical Information Service, Springfield, VA 22161, in paper copy or microfiche form. 
U.S. Department of Commerce

National Institute of Standards and Technology

325 Broadway

Boulder, Colorado 80303-3328

Official Business

Penalty for Private Use, $\$ \mathbf{3 0 0}$ 